\title{
Efficacy of immune checkpoint inhibitors for in-transit melanoma
}

To cite: Nan Tie E, Lai-Kwon J, Rtshiladze MA, et al. Efficacy of immune checkpoint inhibitors for in-transit melanoma. Journal for ImmunoTherapy of Cancer 2020;8:e000440. doi:10.1136/ jitc-2019-000440

ENT and JL-K contributed equally.

Accepted 31 March 2020

Check for updates

(c) Author(s) (or their employer(s)) 2020. Re-use permitted under CC BY-NC. No commercial re-use. See rights and permissions. Published by BMJ.

${ }^{1}$ Peter MacCallum Cancer Centre, Melbourne, Victoria, Australia

${ }^{2}$ Melanoma Institute Australia, North Sydney, New South Wales, Australia

${ }^{3}$ Princess Alexandra Hospital, Woolloongabba, Queensland, Australia

${ }^{4}$ The University of Melbourne, Melbourne, Victoria, Australia

Correspondence to Associate Professor David E Gyorki;

David.Gyorki@petermac.org

Emilia Nan Tie (10) , Julia Lai-Kwon, ${ }^{1}$ Michael Alexander Rtshiladze ${ }^{2}$ Lumine Na, ${ }^{1}$ James Bozzi, ${ }^{2}$ Tavis Read, ${ }^{3}$ Victoria Atkinson, ${ }^{3}$ George Au-Yeung, ${ }^{1}$ Georgina V Long, ${ }^{2}$ Grant A McArthur, ${ }^{1}$ Shahneen Sandhu, ${ }^{1,4}$ Robyn Saw, ${ }^{2}$ Euan Walpole, ${ }^{3}$ Alexander Menzies, ${ }^{2}$ Mark Smithers, ${ }^{3}$ David E Gyorki ${ }^{1,4}$

\section{ABSTRACT}

Background The efficacy of immune checkpoint inhibitors (ICI) in metastatic melanoma is well established. However, there are limited data regarding their efficacy in in-transit melanoma (ITM). This study assessed the efficacy of ICl in patients with ITM.

Methods A retrospective review of patients with ITM commenced on an ICI between March 2013 and February 2018 at three tertiary centers in Australia. Patients were excluded if they had previous or synchronous distant metastases. Overall response rate (ORR), progression-free survival (PFS) and overall survival (OS) were based on a composite of radiological and clinical assessments. Results Fifty-four patients were included: 27 (50\%) female; median age 75 (range 26-94); 12 (22\%) stage IIIB, 40 (74\%) stage IIIC and 2 (4\%) stage IIID; 10 (19\%) BRAF mutant. Forty $(74 \%)$ received single-agent anti-PD-1 (pembrolizumab or nivolumab), $8(15 \%)$ single agent antiCTLA-4 (ipilimumab), 5 (9\%) combination anti-PD-1/antiCTLA-4 (ipilimumab and nivolumab or pembrolizumab) and $1(2 \%)$ combination anti-PD-L1 (atezolizumab) and MEK inhibitor (cobimetinib). The median follow-up was 15 months (2-46).

ORR to ICl was 54\%: 14 (26\%) complete responses; 15 (28\%) partial responses; 9 (17\%) stable disease; $16(30 \%)$ progressive disease. Thirteen (46\%) responders had only one ITM lesion. ORR was $58 \%$ for single-agent anti-PD-1, $38 \%$ for single-agent anti-CTLA4 and 40\% for anti-PD-1/ anti-CTLA-4. The median PFS was 11.7 months (6.6-not reached). 1-year and 2-year PFS were $48 \%$ and $39 \%$, respectively,. Fourteen progressed locoregionally and 11 progressed distantly. The median OS was not reached. 1-year and 2-year OS were $85 \%$ and $63 \%$, respectively. No clinicopathological features were associated with ORR. Conclusions and relevance $\mathrm{ICl}$ produce objective responses in ITM and should be considered in patients with unresectable ITM or disease recurrence.

\section{BACKGROUND AND RATIONALE}

The advent of immune checkpoint inhibitors (ICI) and BRAF and MEK inhibitors has transformed the treatment landscape and prognosis of patients with metastatic melanoma. In seminal studies, pembrolizumab resulted in a 5-year overall survival (OS) of $34 \%$ and the combination of ipilimumab and nivolumab produced a 4-year OS rate of $53 \% .^{12}$ In-transit melanoma (ITM) is defined by the presence of metastases in the superficial lymphatic system more than $2 \mathrm{~cm}$ from the primary lesion but not beyond the regional lymph node basin. Patients with locoregional disease and ITM alone are not well represented in landmark studies.

The natural history of ITM is variable; some tumors have limited systemic metastatic potential and present with multiple locoregional recurrences over years while others rapidly develop distant metastases. Although the 5-year OS for patients with ITM exceeds that of patients with distant metastatic disease (83\%, $69 \%$ and $32 \%$ for stage IIIB, IIIC and IIID disease), a significant percentage of ITM patients experience morbidity from their disease and may eventually develop distant metastases. ${ }^{34}$ Surgical excision has been the mainstay of treatment for ITM. However, there is limited evidence to guide therapy when ITM is either clinically or technically unresectable. Locoregional approaches including topical therapies, intralesional injections, radiotherapy, laser ablation and isolated limb infusion or perfusion (ILI) have demonstrated efficacy. ${ }^{5}$ Intralesional injections with PV-10 (Rose Bengal) or talimogene laherparepvec (T-VEC) produce durable responses, with complete response (CR) rates of $26 \%$ and $16 \%$, respectively, in patients with stage IIIB-IV disease. ${ }^{6} 7$ Similarly, ILI has demonstrated a CR rate of $38 \%$ with a median duration of response of 13 months. ${ }^{8}$ However, responses in these studies should be interpreted with care as majority of were small single arm studies or case series.

Systemic therapies are increasingly used in unresectable stage III melanoma including patients with ITM. ICIs have the potential to alter the natural history of ITM by preventing the development of further locoregional or distant metastases while limiting the morbidity associated with certain 
Table 1 Baseline characteristics stratified by $\mathrm{ICl}$

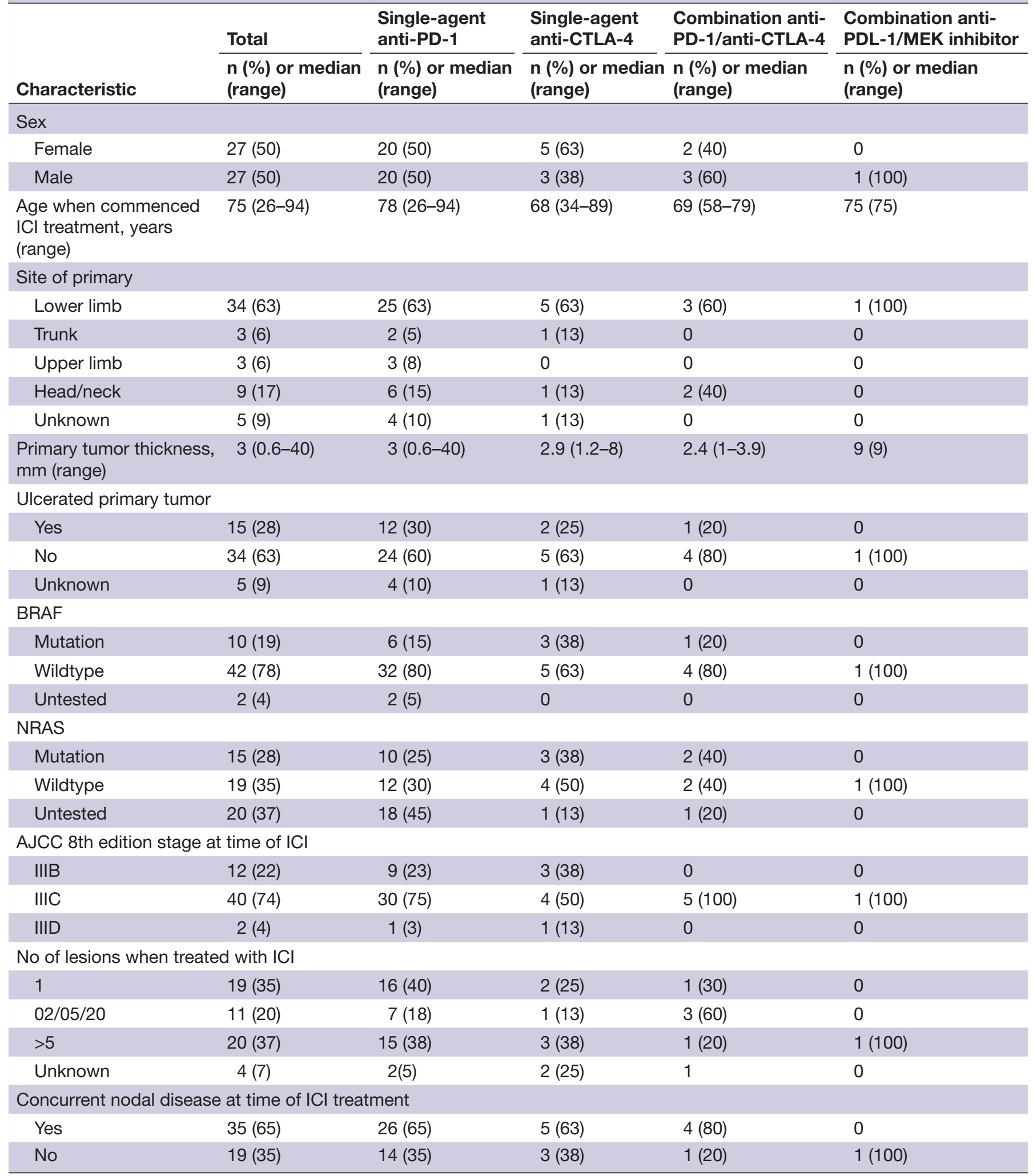

AJCC, American Joint Committee on Cancer; ICI, immune checkpoint inhibitors.

locoregional therapies. However, limited data exist ICI efficacy for ITM. Registration studies of ICI for metastatic melanoma did not prespecify ITM as a subgroup for analysis. These patients were included in the patient group with unresectable stage III disease which accounted for less than $10 \%$ of patients enrolled. ${ }^{129-11}$ Where this subgroup was analyzed retrospectively, conclusions were limited due to small patient numbers. ${ }^{129-11}$ The aim of 
Table 2 Overall response to immune checkpoint-inhibitors in patients with in-transit melanoma metastases

\begin{tabular}{|c|c|c|c|c|c|}
\hline & Total & $\begin{array}{l}\text { Single-agent } \\
\text { anti-PD-1 }\end{array}$ & $\begin{array}{l}\text { Single-agent } \\
\text { anti-CTLA-4 }\end{array}$ & $\begin{array}{l}\text { Combination anti- } \\
\text { PD-1/anti-CTLA-4 }\end{array}$ & $\begin{array}{l}\text { Combination anti- } \\
\text { PDL-1/MEK inhibitor }\end{array}$ \\
\hline Overall response & $\mathbf{N}(\%)$ & $\mathrm{N}(\%)$ & $\mathbf{N}(\%)$ & N (\%) & N (\%) \\
\hline Overall & $54(100)$ & 40 (100) & $8(100)$ & $5(100)$ & $1(100)$ \\
\hline $\mathrm{CR}$ & $14(26)$ & $12(30)$ & $1(13)$ & $1(20)$ & 0 \\
\hline PR & $15(28)$ & $11(28)$ & $2(25)$ & $1(20)$ & $1(100)$ \\
\hline SD & $9(17)$ & $4(10)$ & $3(38)$ & $2(40)$ & 0 \\
\hline PD & $16(30)$ & $13(33)$ & 2 (25) & $1(20)$ & 0 \\
\hline
\end{tabular}

CR, complete response; PD, progressive disease; PR, partial response; SD, stable disease.

this study was to confirm the response to ICI in patients with only ITM.

\section{METHODS}

We conducted a retrospective review of patients with of ITM treated with ICI across three tertiary hospitals in Australia, following individual institutional ethics committee approval. Patients with local recurrence or satellites alone were not included, but patients with satellites together with other ITM were included. Patients were excluded if they had previous or synchronous metastatic disease. Data regarding patient demographics, clinicopathological staging at diagnosis and at commencement of ICI and prior regional therapies were collected from the medical record.

Overall response rate (ORR) was defined as CR plus partial response (PR) based on a composite of radiological and clinical assessments. PR was defined as clinical disease reduction of at least $50 \%$. ITMs are often non-evaluable by response evaluation criteria in solid tumors (RECIST). Progression-free survival (PFS) was measured from ICI commencement to radiological or clinical progression or death. OS was measured from ICI commencement to death. PFS and OS were estimated

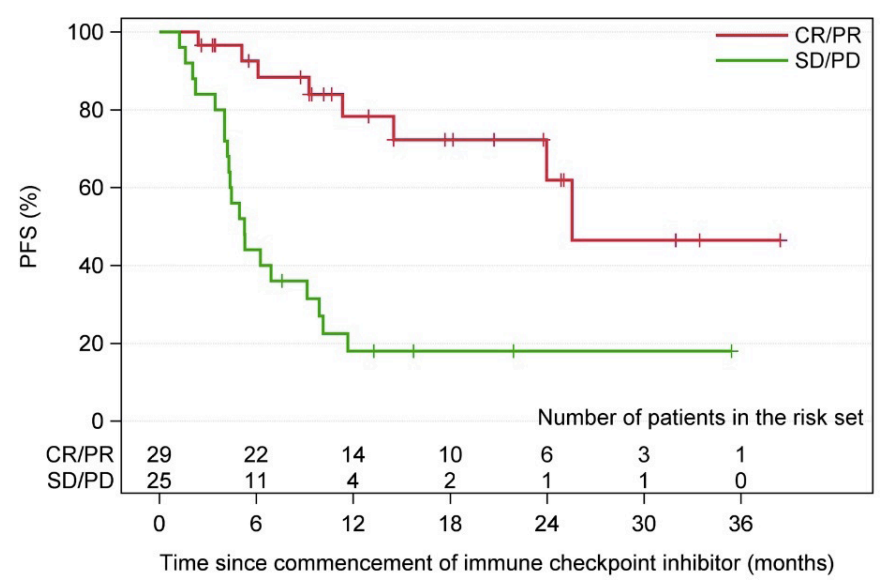

Figure $1 \mathrm{CR}$, complete response; PD, progressive disease; PFS, progression free survival; PR, partial response; SD, stable disease. using the Kaplan-Meier method and survival distributions were compared using a log-rank test.

The association between ORR and potential clinical and pathological factors were assessed using Fisher's exact test (two sided) if nominal variables (ulceration, BRAF status, previous intralesional/ILI therapy), Exact Cochran-Armitage Trend test (one sided) if ordinal variables (number of lesions, stage at commencement) and Wilcoxon test (two sided) if continuous variables (first disease free interval, ITM disease-free interval). Analyzes were carried out using SAS (V.9.4; SAS Institute).

\section{RESULTS}

Fifty-four patients received ICI for unresectable ITM between March 2013 and February 2018. Baseline characteristics are shown in table $1 ; 10$ patients $(19 \%)$ had BRAF mutant melanoma, $11(20 \%)$ patients received ICI as first-line treatment for their ITM, $6(11 \%)$ had prior ILI treatment and $17(31 \%)$ had prior intralesional therapies (T-VEC: 2 (4\%) or PV-10: 15 (28\%)). Forty patients (74\%) received single-agent PD-1 inhibitor (pembrolizumab or nivolumab), eight (15\%) single-agent anti-CTLA-4 (ipilimumab), five (9\%) combination anti-PD-1/anti-CTLA-4 (ipilimumab and nivolumab or pembrolizumab) and one (2\%) combination anti-PDL-1 (atezolizumab) and MEK inhibitor (cobimetinib). Other treatment modalities were used in combination with ICI in some instances: three $(6 \%)$ patient received radiotherapy, one (2\%) patient had intralesional injection and one $(2 \%)$ patient had topical therapy.

The ORR to ICI was 54\%: 14 (26\%) CR; 15 (28\%) PR; 9 $(17 \%)$ stable disease (SD); $16(30 \%)$ progressive disease (PD). The ORR was 58\% for single agent anti PD-1, 38\% for single-agent anti-CTLA- 4 and 40\% for anti-PD-1/antiCTLA-4 (table 2). Ulceration, BRAF status, number of lesions, prior treatments (ILI or intralesional injection) and stage also had no significant association with ORR.

The median duration of follow-up was 15.1 months (95\% CI 2 to 46). Twenty-five patients progressed (48\%): $14(56 \%)$ progressed locoregionally and 11 (44\%) developed distant metastases. The median PFS was 11.7 months (6.3-not reached). One-year and 2-year PFS were 
$48 \%(95 \%$ CI $33 \%$ to $61 \%)$ and $39 \%$ (95\% CI $23 \%$ to $55 \%$ ), respectively (figure 1). Further 2-year PFS for anti PD-1, anti-CTLA-4 and anti-PD-1/anti-CTLA-4 was 30\%, $50 \%$ and $80 \%$, respectively.

The median OS was not reached, three $(6 \%)$ patients died. One-year and 2-year OS rate were $85 \%(95 \%$ CI $71 \%$ to 93 ) and $63 \%$ (95\% CI $43 \%$ to $77 \%$ ), respectively. At 2 years, the OS for patients with an overall response was $85 \%$ (95\% CI $50 \%$ to $96 \%$ ) compared with $40 \%$ (95\% CI $16 \%$ to $64 \%$ ) in those who did not.

\section{DISCUSSION}

This study demonstrates that single agent and combination ICI produces durable responses in ITM similar to rates seen in patients with stage IV melanoma. The 2-year PFS of $39 \%$ and 2-year OS of $63 \%$ are consistent with landmark survival data from registration studies for ICI in unresectable stage III and stage IV melanoma (pembrolizumab resulted in a 5-year OS of $34 \%$ study and ipilimumab/nivolumab a 4-year OS rate of 53\%). ${ }^{2}{ }^{9-11}$ Objective measurements of responses are challenging in the ITM population as the majority of patients have disease which is non-evaluable by RECIST. Thus, this study relied on subjective clinician assessed responses as well as radiological responses, a significant limitation. The lower than expected response rate in the anti-PD-1/ anti-CTLA-4 group may be due to the small sample size and higher relative disease burden.

While ICI is clearly an effective treatment for ITM, there is minimal data to guide how ICI could be optimally integrated with other commonly used locoregional therapies for ITM. For patients with slowly progressing, isolated or low volume ITM, surgical resection remains a standard of care, however, for patients with rapidly progressive disease the selection of locoregional therapies, ICI or a combination of both is unclear. A phase $1 \mathrm{~b} / 2$ study evaluating the combination of PV-10 and pembrolizumab in 23 patients with stage IIIB-IV M1c melanoma demonstrated a CR rate of $77 \% .{ }^{12}$ Further, a phase II study of 18 patients with stage IIIB-IV melanoma (previous treatment not reported) examined the efficacy of ILI in combination with ipilimumab. This study demonstrated a CR rate of $65 \%$ and a 1-year PFS of $57 \% .{ }^{13}$ Therefore, combinations of locoregional therapy and ICI may produce higher ORR and more durable responses than either treatment in isolation and warrants further examination in larger studies; such as MASTERKEY-265 comparing pembrolizumab with or without T-VEC (NCT02263508). In our retrospective analysis, prior treatment with ILI or intralesional injections was not associated with higher response rates to subsequent ICI therapy.

\section{CONCLUSION}

This multi-institutional study provides the largest evidence base of durable responses to ICI in patients with ITM, demonstrating this to be an effective treatment in patients with with unresectable ITM or disease recurrence despite locoregional therapies. Prospective studies specifically targeting this unique population are needed to confirm the efficacy of ICI and to determine optimal sequencing of treatment and potential locoregional combinations.

Correction notice Since the online publication of this article, the authors noticed that the middle initial for author 'Georgina V Long' was missing. This has been corrected.

Twitter Julia Lai-Kwon @julialaikwon and David E Gyorki @davidgyorki

Acknowledgements Melanoma Research Victoria, Queensland Melanoma Project, Melanoma Institute Australia.

Contributors ENT: Conceptualization, data collection and curation, project administration, writing — original draft. JL-K: Conceptualization, writing—original draft. MAR: data collection and curation, writing — review and editing. LN: statistical analysis. JB: data collection and curation. TR: data collection and curation. VA: writing — review and editing. GA-Y: writing—review and editing. GL: writing— review and editing. GAM: writing — review and editing. SS: writing — review and editing. RS: Conceptualization, supervision, data collection and curation, writingreview and editing. EW: writing — review and editing. AM: Conceptualization, supervision, data collection and curation, writing — review and editing. MS: writing — review and editing. DEG: Conceptualization, project administration, resources, supervision, writing —review and editing.

Funding The authors have not declared a specific grant for this research from any funding agency in the public, commercial or not-for-profit sectors.

Competing interests VA-Advisory Boards: BMS, MSD, Merck, Novartis, Roche, Pierre Fabre. Travel Support: BMS, Onco-sec. Speakers Fee: BMS, MSD, Merck, Novartis, Roche. GL — consultant advisor to Aduro, Amgen, Array pharmaceutical, BMS, MSD, Novartis, Roche, Pierre-Fabre. SS—consultant advisor to Astra Zeneca, MSD, Novartis, BMS, Amgen, Roche and Janssen. RS—Advisory board MSD, Novartis and speaking honoraria BMS. AM—consultant advisor to BMS, MSD, Novartis, Roche, Pierre-Fabre. DEG—advisory board and received honoraria from Amgen and from Provectus.

Patient consent for publication Not required.

Ethics approval Individual institutional ethics committee approval was obtained.

Provenance and peer review Not commissioned; externally peer reviewed.

Data availability statement Data are available on reasonable request. The datasets generated during and/or analysed during the current study are not publicly available due to patient confidentiality but are available from the corresponding author on reasonable request.

Open access This is an open access article distributed in accordance with the Creative Commons Attribution Non Commercial (CC BY-NC 4.0) license, which permits others to distribute, remix, adapt, build upon this work non-commercially, and license their derivative works on different terms, provided the original work is properly cited, appropriate credit is given, any changes made indicated, and the use is non-commercial. See http://creativecommons.org/licenses/by-nc/4.0/.

ORCID iD

Emilia Nan Tie http://orcid.org/0000-0003-2728-7526

\section{REFERENCES}

1 Hamid O, Robert C, Daud A, et al. Five-Year survival outcomes for patients with advanced melanoma treated with pembrolizumab in KEYNOTE-001. Ann Oncol 2019;30:582-8.

2 Hodi FS, Chiarion-Sileni V, Gonzalez R, et al. Nivolumab plus ipilimumab or nivolumab alone versus ipilimumab alone in advanced melanoma (CheckMate 067): 4-year outcomes of a multicentre, randomised, phase 3 trial. Lancet Oncol 2018;19:1480-92.

3 Gershenwald JE, Scolyer RA, Hess KR, et al. Melanoma staging: evidence-based changes in the American joint Committee on cancer eighth edition cancer staging manual. CA Cancer J Clin 2017;67:472-92.

4 Read RL, Haydu L, Saw RPM, et al. In-Transit melanoma metastases: incidence, prognosis, and the role of lymphadenectomy. Ann Surg Oncol 2015;22:475-81. 
5 Read T, Lonne M, Sparks DS, et al. A systematic review and metaanalysis of locoregional treatments for in-transit melanoma. J Surg Oncol 2019;119:887-96.

6 Thompson JF, Agarwala SS, Smithers BM, et al. Phase 2 study of intralesional PV-10 in refractory metastatic melanoma. Ann Surg Oncol 2015;22:2135-42.

7 Andtbacka RHI, Kaufman HL, Collichio F, et al. Talimogene Laherparepvec improves durable response rate in patients with advanced melanoma. J Clin Oncol 2015;33:2780-8.

8 Kroon HM, Moncrieff M, Kam PCA, et al. Outcomes following isolated limb infusion for melanoma. A 14-year experience. Ann Surg Oncol 2008;15:3003-13.

9 Hodi FS, O'Day SJ, McDermott DF, et al. Improved survival with ipilimumab in patients with metastatic melanoma. $N$ Engl $J$ Med 2010;363:711-23.
10 Robert C, Thomas L, Bondarenko I, et al. Ipilimumab plus dacarbazine for previously untreated metastatic melanoma. $N$ Engl $J$ Med 2011;364:2517-26.

11 Robert C, Ribas A, Hamid O, et al. Long-Term outcomes in patients (PTS) with advanced melanoma treated with pembrolizumab (Pembro): 4-year overall survival (OS) results from KEYNOTE-001. Munich, Germany: 7th European Post-Chicago Melanoma/Skin Cancer Meeting, 2017.

12 Agarwala SS, Ross MI, Zager JS, et al. Phase 1B study of PV-10 and anti-PD-1 in advanced cutaneous melanoma. JCO 2019;37:9559

13 Ariyan CE, Lefkowitz RA, Panageas K, et al. Safety and clinical activity of combining systemic ipilimumab with isolated limb infusion in patients with in-transit melanoma. JCO 2014;32:9078. 
Correction: Efficacy of immune checkpoint inhibitors for intransit melanoma

Nan Tie E, Lai-Kwon J, Rtshiladze MA, et al. Efficacy of immune checkpoint inhibitors for in-transit melanoma. J Immunother Cancer 2020;8:e00440. doi: 10.1136/jitc-2019000440

Since the online publication of this article, the authors noticed that the middle initial for author 'Georgina V Long' was missing. This has now been corrected.

Open access This is an open access article distributed in accordance with the Creative Commons Attribution Non Commercial (CC BY-NC 4.0) license, which permits others to distribute, remix, adapt, build upon this work non-commercially, and license their derivative works on different terms, provided the original work is properly cited, appropriate credit is given, any changes made indicated, and the use is non-commercial. See: http://creativecommons.org/licenses/by-nc/4.0/.

(C) Author(s) (or their employer(s)) 2020. Re-use permitted under CC BY-NC. No commercial re-use. See rights and permissions. Published by BMJ.

J Immunother Cancer 2020;8:e000440corr1. doi:10.1136/jitc-2019-000440corr1

D) Check for updates 